\title{
Compositional interpretation of the geometric albedo of asteroids
}

\section{Solar phase effects}

\author{
J. M. Carvano \\ Observatório Nacional (COAA), rua Gal. José Cristino 77, São Cristóvão, CEP20921-400 Rio de Janeiro RJ, Brazil \\ e-mail: carvano@on.br
}

Received 23 January 2008 / Accepted 19 March 2008

ABSTRACT

\begin{abstract}
Aims. In this first paper we investigate the dependence of the geometric albedo on the phase function of the particles that cover it, and derive the expected geometric albedo of bodies for a given mineralogy, taking into account the constraints imposed by the observed phase functions of the asteroids.

Methods. A genetic fitting algorithm is used to fit Hapke integral phase functions to Lumme-Bowell integral phase functions described by values of the slope parameter $G$ of the IAU HG system. The resulting geometric albedo of laboratory samples are then compared to the observed values of asteroids with assumed similar mineralogy.

Results. Because of the weak dependence of $\bar{\theta}$ on the integral phase functions it is not possible to find a unique set of Hapke parameters that fit the Lumme-Bowell function for a given value of $G$, at least for phase angles $<60^{\circ}$. Instead, unique solutions can be found if we leave $\bar{\theta}$ as a free parameter. It is shown that the laboratory derived scattering parameters in general fail to match the geometric albedo and slope parameter of asteroids of presumed equal mineralogy. It is also shown that a given value of the single scatter albedo can lead to very different values of $p_{\mathrm{v}}$, depending on $G$ and $\bar{\theta}$. The methodology developed is used to compare the observed $p_{\mathrm{v}}$ and $G$ of the asteroids (4) Vesta and (21) Lutetia with laboratory measurements of materials with suposedly similar compositions. As expected, it is found that the albedo and slope parameter of Vesta are compatible with measurements of unweathered terrestrial basalts with grain sizes $<=250 \mu \mathrm{m}$. The albedo and slope parameter of Lutetia are found to be compatible with samples of the Allende CV3 meteorite for grain sizes $<500 \mu \mathrm{m}$. The routines that allow the conversion between $w$ and $p_{\mathrm{V}}$ (and vice-versa) are available at http://funk. on. br/ carvano/albedo/albedo.html
\end{abstract}

Key words. minor planets, asteroids

\section{Introduction}

The geometric albedo is a measure of the fraction of the incoming radiation on a given bandpass (usually the visible) that is reflected by a body. Among the many different ways of defining "albedo", this is the most convenient for remote sensing purposes and hence it is widely used to characterize the intrinsic reflectivity of solar system bodies. The geometric albedo has been commonly used as an auxiliary parameter in attempts to determine the composition of those bodies. Examples of this are the still widely used taxonomies of Tholen \& Barucci (1989). The geometric albedo in some instances has also been used to scale reflectance spectra in compositional analysis involving radiative transfer methods (i.e. Clark et al. 2004). Such uses are deemed necessary since the absolute reflectance of a material is an important compositional discriminant that cannot be directly assessed from the spectra of disk-integrated sources. However, this assumes that the geometric albedo can be directly related to the volumetric single scatter albedo $(w)$ of the particles that coat the body. This assumption is not necessarily true, since the geometric albedo can be significantly influenced by the packing characteristics of the surface (which determine other scattering parameters of the medium) and the shape of the body. Therefore, simply using the geometric albedo to make compositional inferences might lead to misleading results.

The effects of the scattering parameter on the geometric albedo are modeled by Hapke's formulation (Hapke 1993), which describe the scattering properties using a minimum of
5 parameters. Since some of the scattering parameters depend on the packing of the material and lab conditions may not reproduce what really happens on the surface of an airless, low gravity body, it is very likely that the scattering parameters measured in the lab may not be adequate to describe the surface of asteroids. Indeed, it is known that asteroids tend to have asymmetry parameters $<0$ while lab materials generally yield asymmetry parameters $>0$ (Helfenstein \& Veverka 1989). Thus, using lab scattering parameters to estimate the resulting geometric albedo of a given material can also be misleading.

A more sound approach would be to take into account the solar phase behavior of the asteroids when trying to estimate $w$ from the geometric albedo. It is in principle possible to derive the scattering parameter of asteroids by fitting solar phase curves, but this needs observations over a comprehensive range of solar phase angles, which is not available for most asteroids. Also, fitting multiple parameter lead to uncertainties, since multiple equally good fits may exist (i.e., Domingue \& Hapke 1989; Helfenstein \& Veverka 1989). A more widely available indicator of the solar phase behavior of the asteroids is given by the HG system that was adopted by the IAU in 1985 (Bowell et al. 1989). This system uses an empirical integral solar phase function from the Lumme-Bowell formulation that is completely determined by the slope parameter $G$. It was found to reproduce well the variation of brightness of asteroids as a function of solar phase angle, but the parameter $G$ lacks a direct physical interpretation in terms of the scattering properties of 
the surfaces. Verbiscer \& Veverka (1995) derived a way to convert the HG system to Hapke parameters. Their approach assumes a fixed value for the mean surface slope. However, recent work (Sakai \& Nakamura 2005) highlights the influence of this parameter, which is related to the porosity of the surface, on reflectance measurements. Also, there is evidence in the literature that the surface porosity of airless bodies can be fairly high (Carvano et al. 2007).

The aim of this work is to analyze the effects of surface packing and shape on the geometric albedo and to provide a framework in which the compositional inferences based on the geometric albedo can be made in a way that is consistent with other observational parameters of the body. This first paper deals with solar phase effects that are induced by the packing characteristics of surfaces. To this end I analyze in Sect. 2 the existence of multiple solutions to the problem of obtaining the Hapke parameter that best fits the integral phase functions generated by any given $G$, assuming a single-lobed Heyney-Greenstein function as the particle phase function. The result of this exercise is a method that allows one to determine the the geometric albedo scattering parameter, $\left(\xi, B_{0}, h, p_{\mathrm{v}}\right)$, as a function of $w, G$ and $\bar{\theta}$, or, conversely, $\left(\xi, B_{0}, h, w\right)$ as functions of $p_{\mathrm{v}}, G$ and $\theta$. In Sect. 3 this formulation is then used to compared the predicted geometric albedo of laboratory samples to observations of real asteroids. General conclusions are drawn in Sect. 4. The influences of the shape in the geometric albedo will be analyzed in a forthcoming paper.

\section{Relating the geometric albedo to the slope parameter $\mathbf{G}$}

The geometric albedo of a spherical body is defined as the ratio of the brightness of a body at zero solar phase angle to the brightness of a perfect Lambertian disk of the same radius and at the same distance as the body, but illuminated and observed perpendicularly (Hapke 1993, H93 from here on). For a spherical body this is expressed by Eq. (1):

$$
\begin{aligned}
p_{\lambda}\left(w_{\lambda}, \bar{\theta}\right)= & \left.\frac{w_{\lambda}}{8}\left\{1+B_{0}\right] P\left(g=0, \xi_{1}, \ldots\right)-1\right\} \\
& +\mathcal{U}\left(w_{\lambda}, \bar{\theta}\right) \frac{r_{0}}{2}\left(1+\frac{r_{0}}{3}\right) \\
r_{0}= & \frac{2}{1+\sqrt{1-w_{\lambda}}}-1
\end{aligned}
$$

where $g$ is the solar phase angle, $w_{\lambda}$ is the volumetric single scatter albedo, $B_{0}$ is the amplitude of the opposition effect, $P$ is the volumetric phase function, $\bar{\theta}$ is the mean slope angle of the surface and $\mathcal{U}$ is a correction function for macroscopic roughness, which can be approximated by:

$$
\mathcal{U}\left(w_{\lambda}, \bar{\theta}\right)=1-\left(0.048 \bar{\theta}+0.0041 \bar{\theta}^{2}\right) r_{0}-\left(0.33 \bar{\theta}-0.0049 \bar{\theta}^{2}\right) r_{0}^{2},
$$

where $\bar{\theta}$ is given in radians (H93). Presently there is no theoretically satisfactory derivation for the volumetric phase function. Instead a number of empirical functions have been adopted in the literature, which depend on one or more parameters $\xi_{i}$, whose physical significance depends on the form of the adopted function. For example, a single-lobed Henyey-Greenstein function is fully defined with only the asymetry parameter $\xi$.

Thus, the geometric albedo of a spherical body depends on at least four scattering parameters of the material on its surface: the volumetric single scatter albedo $\left(w_{\lambda}\right)$, which depends on the composition and size distribution of the particles, the mean slope angle $(\bar{\theta})$, which depend on the particle shape, particle size distribution, filling factor and smoothness of the surface, plus the solar phase parameter(s) and the amplitude of the opposition effect $B_{0}$, which depends both on the composition and size of the particles and on the texture of the surface.

Other quantity of interest is the integral phase function, which is the relative brightness of the entire body seen at a given phase angle, normalized to its brightness at zero phase angle. For a spherical body we have (H93):

$$
\begin{aligned}
\Phi(g)= & \mathcal{K}(g, \bar{\theta}) \frac{r_{0}}{2 p_{\lambda}}\left\{\left[\frac{(1+\sqrt{1-w})^{2}}{4}\right.\right. \\
& \times\left\{\left[1+B\left(g ; B_{0}, h\right)\right] P\left(g ; \xi_{1}, \ldots\right)-1\right\} \\
& \left.+\left(1-r_{0}\right)\right]\left[1-\sin \frac{g}{2} \tan \frac{g}{2} \ln \left(\cot \frac{g}{4}\right)\right] \\
& \left.+\frac{4}{3} r_{0} \frac{\sin g+(\pi-g) \cos g}{\pi}\right\} \\
B(g)= & \frac{B_{0}}{1+\left(\frac{1}{h} \tan \frac{g}{2}\right)}
\end{aligned}
$$

where $h$ is the angular width of the opposition effect, which depends on the texture of the surface, and $\mathcal{K}$ is the correction for the macroscopic rugosity, which for $g<60^{\circ}$ can be approximated by:

$$
\mathcal{K}(g, \bar{\theta})=\exp \left\{-0.32 \bar{\theta}\left[\tan \bar{\theta} \tan \frac{g}{2}\right]^{2}-0.52 \bar{\theta} \tan \bar{\theta} \tan \frac{g}{2}\right\} .
$$

The integral phase function in Hapke formulation thus depends on a minimum of five scattering parameters. In principle all parameters can be derived fitting the observed magnitude at several phase angles, normalized to the geometric albedo. A problem noted here is that, unless observations at phase angles greater than $90^{\circ}$ are available, the fit produces non-unique solutions (Domingue \& Hapke 1989; Helfenstein \& Veverka 1989). An alternative way to describe the integral phase function is the Lumme-Bowell model which was adopted by the IAU (Bowell et al. 1989). In this model the integral phase function of the asteroids is represented by an empirical function that is fully specified by the slope parameter $G$ :

$\Phi(g)=(1-G) \Phi_{1}(g)+G \Phi_{2}(g)$

(see Bowell et al. 1989) for the definition of $\Phi_{i}$ ). To obtain a transformation of the IAU system to Hapke parameters. Verbiscer \& Veverka (1995) used Eq. (7) to generate normalized magnitudes as functions of solar phase angle for a grid o values of $G$ and $p_{\mathrm{v}}$ and fitted them with the phase function described by Eq. (4) using a one-lobed Henyey-Greenstein function and keeping $\bar{\theta}=20^{\circ}$. In this way the were able to obtain coefficients of polynomial series that allowed to estimate the scattering parameters $\left(w_{\mathrm{v}}, \xi, B_{0}, h\right)$ as functions of $p_{\mathrm{v}}$ and $G$. The procedure adopted in the present work is slightly different: the Hapke integral phase function is fitted directly to the empirical phase function of Lumme-Bowell. For each $G$ the Lumme-Bowell integral phase function was calculated and the fit was performed keeping $w$ fixed. This yields the scattering parameters $\left(\xi, B_{0}, h, \bar{\theta}\right)$ as functions of $w_{\mathrm{v}}$ and $G$, and these parameters can then be inserted in Eq. (1) to yield $p_{\mathrm{v}}$ as a function of $w_{\mathrm{v}}$ and $G$. It is then possible to numerically invert the functions in order to obtain the scattering parameters as a function of $p_{\mathrm{V}}$ and $G$. 


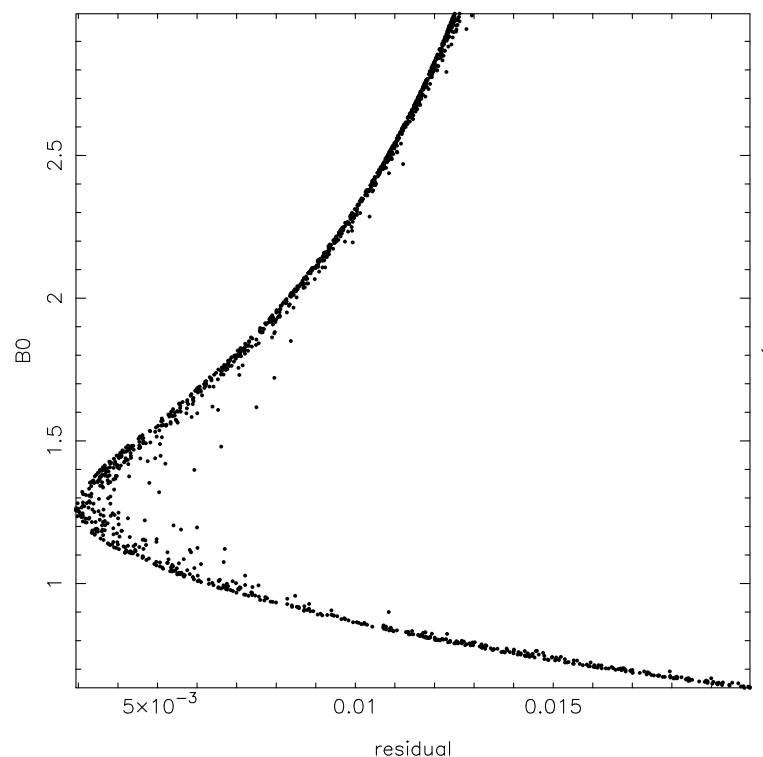

(a)

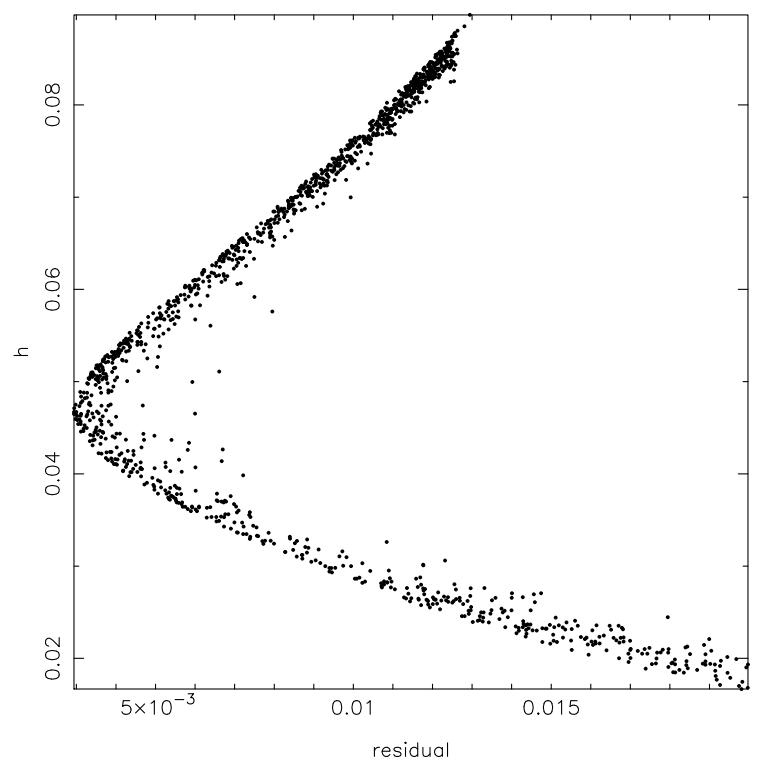

(c)

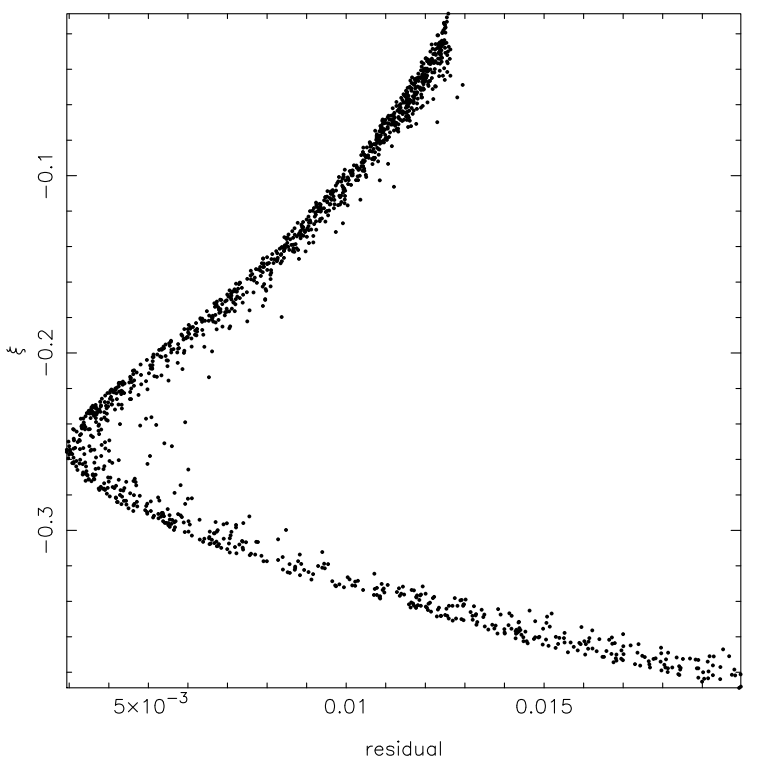

(b)

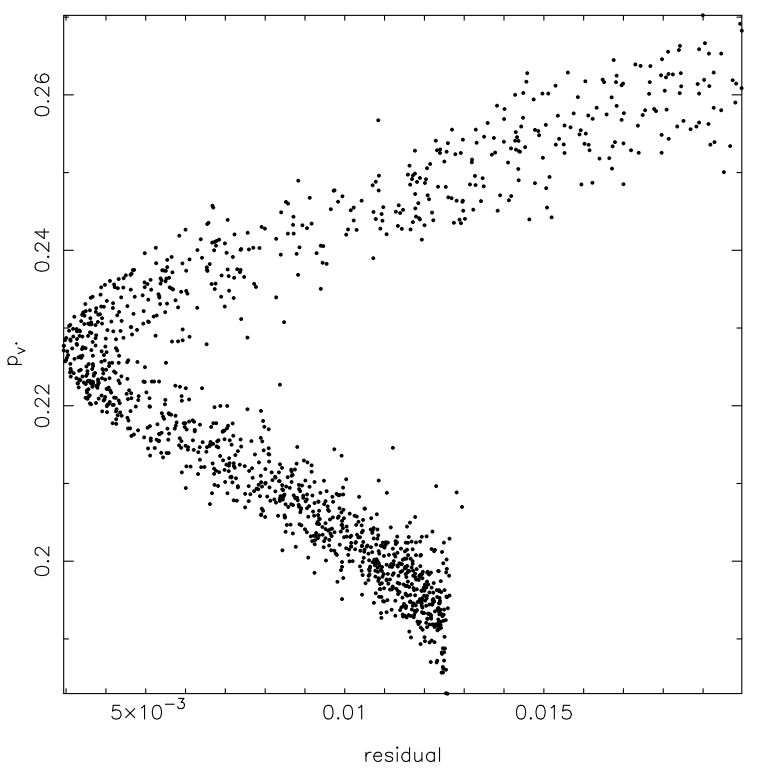

(d)

Fig. 1. Results of the genetic fitting algorithm for $B_{0}, \xi, h$ and the corresponding geometric albedo versus the residual of the fit. In this run $G=0.32$, $w=0.34$ and the macroscopic roughness parameter was kept fixed at $\bar{\theta}=20^{\circ}$.

In order to probe the existence of multiple solutions I initially used a genetic fitting algorithm set to perform deterministic crowding. Genetic algorithms are general purpose, parallel search procedures that are based upon genetic and evolutionary principles (Goldberg 1989; Holland 1992). Determinist crowding (Mahfoud 1995) is a mode of operation were convergence is delayed to force the genetic algorithm to preserve most (possibly all) good solutions found during the evolution of the population.

As an initial verification, I attempted to reproduce the test case of Verbiscer and Veverka. With the mean surface slope fixed at $\bar{\theta}=20^{\circ}$ and with the single scatter albedo set to their best fit value $w_{\mathrm{v}}=0.34$, I attempted to fit the Lumme-Bowell integral phase function for $G=0.32$ in the solar phase range where Eq. (2) is valid $\left(0-60^{\circ}\right)$. I allowed the remaining free parameters to vary within the intervals $B_{0} \in[0,5], h \in[0.01,0.1]$ and $\xi \in$ $[-2,2]$. Results are shown in Fig. 1 as plots of the fitted values
Table 1. Comparison with previous works.

\begin{tabular}{cccc}
\hline \hline Parameter & Helfenstein et al. & Verbiscer \& Veverka & This work \\
\hline$w_{\mathrm{v}}$ & 0.32 & 0.34 & $0.34^{*}$ \\
$p_{\mathrm{v}}$ & $0.23^{*}$ & $0.23^{*}$ & 0.227 \\
$\bar{\theta}$ & $20^{\circ}$ & $20^{\circ}$ & $20^{\circ}$ \\
$h$ & 0.06 & 0.05 & 0.046 \\
$B_{0}$ & 1.2 & 1.2 & 1.256 \\
$\xi$ & -0.26 & -0.25 & -0.255 \\
\hline
\end{tabular}

and the calculated $p_{\mathrm{v}}$ as functions of the residuals of the fit after 500 generations (a total population of 2000 individuals was used). The plots show that as the residual of the fit decreases all the free variables converge to a single solution, and as is shown in Table 1 the best-fit solution is in good agreement with the values of Verbiscer \& Veverka (1995) and Helfenstein et al. (1994). 


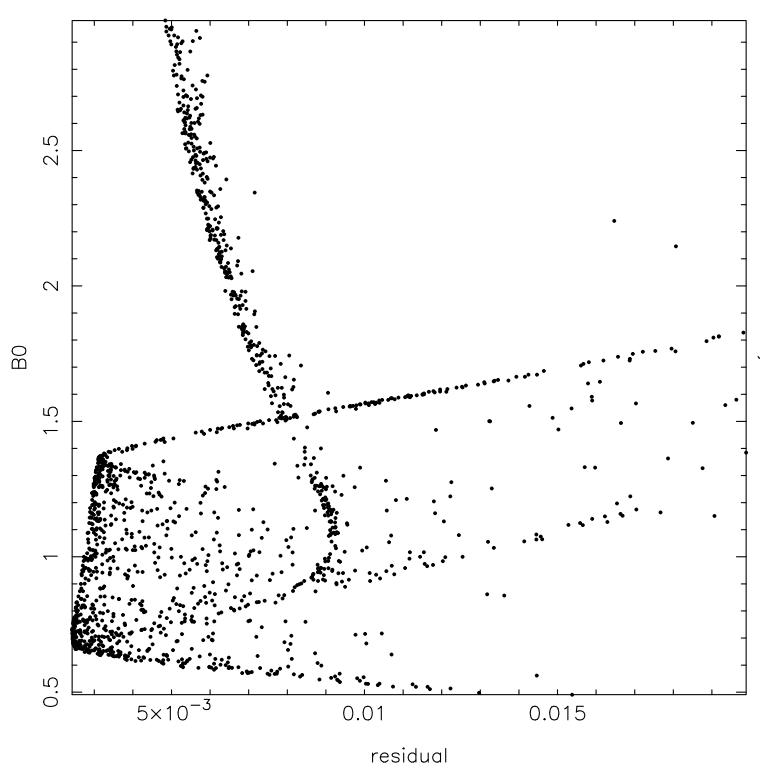

(a)

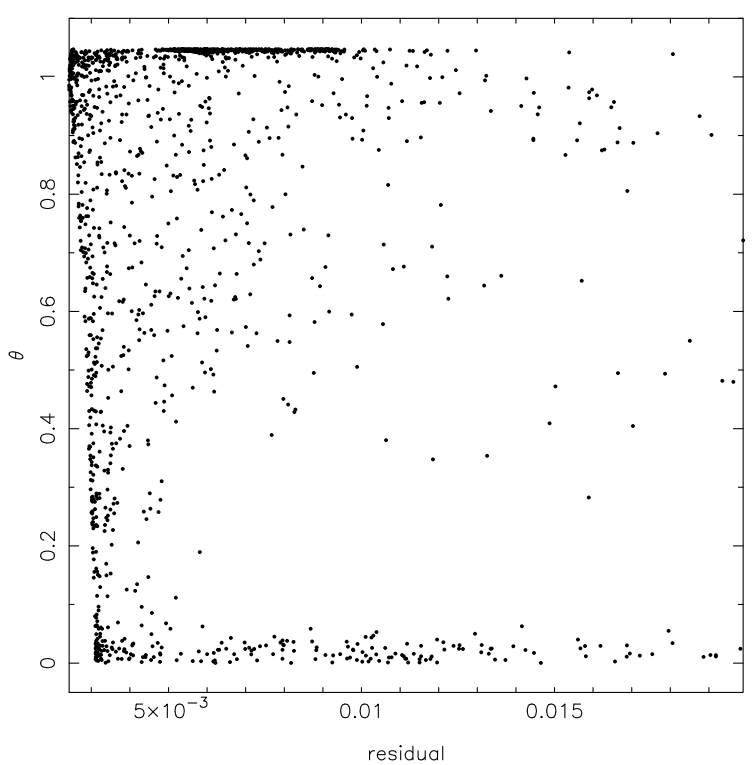

(c)

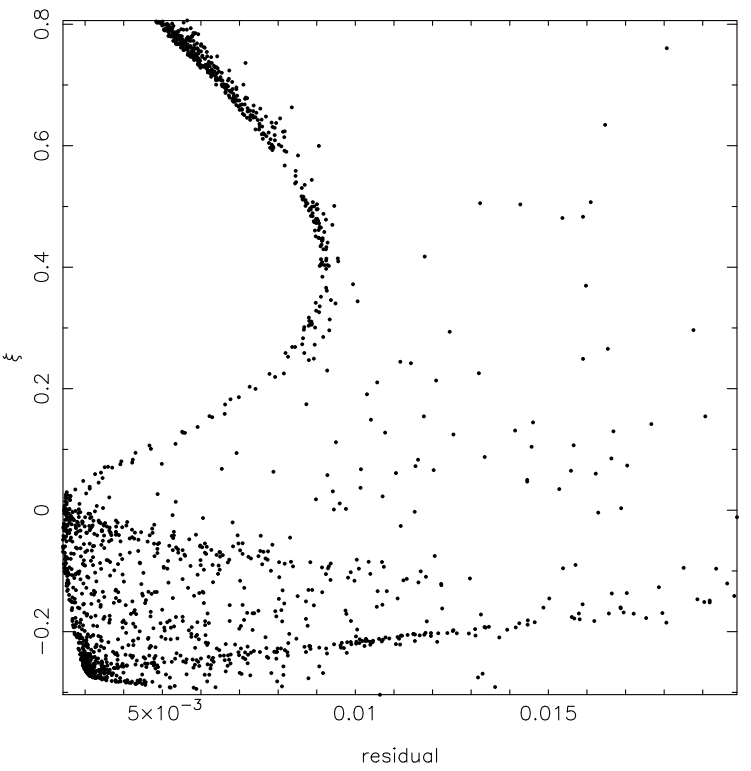

(b)

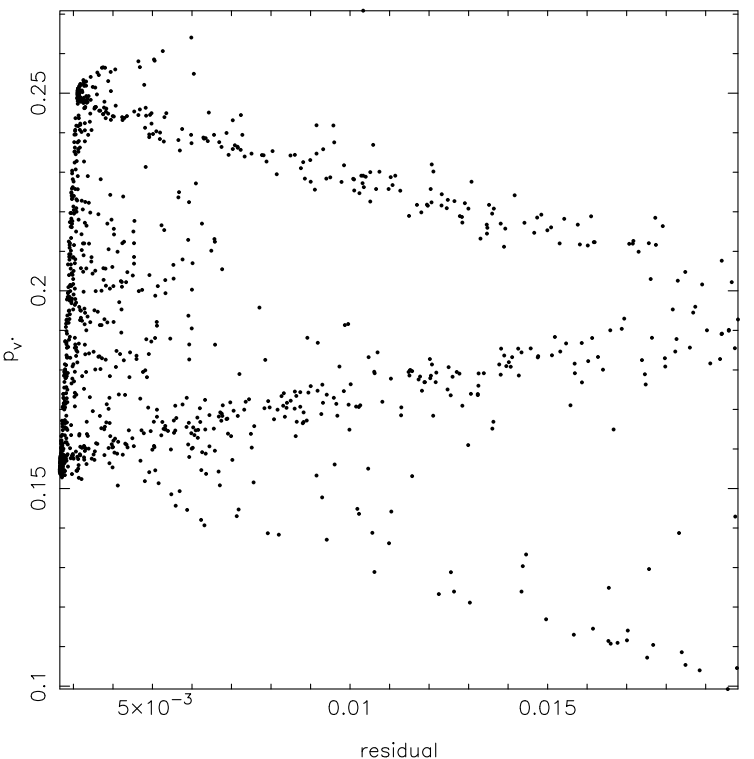

(d)

Fig. 2. Results of the genetic fitting algorithm for $B_{0}, \xi, \bar{\theta}$ and the corresponding geometric albedo versus the residual of the fit. In this run $G=0.32, w=0.34$ and the macroscopic roughness parameter was kept fixed at $\bar{\theta}=20^{\circ}$.

Next I investigate the solution space when $\bar{\theta}$ is left free to vary. For the same values of $G$ and $w_{\mathrm{v}}$ I fixed $h$ at the best fit value of the previous run and let the system evolve again for 500 generations, with the same population, $\bar{\theta} \in\left[0^{\circ}, 60^{\circ}\right]$ and the other free variables in the same interval as in the previous calculations. Results are show in Fig. 2. Here the situation seems much more complex. From Fig. $2 \mathrm{c}$ it can be seen that $\bar{\theta}$ remains essentially unconstrained up to very low residuals, then increases rapidly close to the highes values allowed during the fit (best fit value for $\bar{\theta}=57^{\circ}$ ). This undetermination of $\bar{\theta}$ has a clear impact on the other parameters, which also exhibit a considerable, albeit constrained, variation close to the best solution. Figure 3 shows how the variation of $\bar{\theta}$ affects the other parameters and $p_{\mathrm{V}}$ in the vicinity of the best solution.

The fact that $\bar{\theta}$ cannot be adequately constrained using measurements with $g<90^{\circ}$ is well established in the literature
(Domingue \& Hapke 1989), and the its final convergence is most likely induced by the fitting algorithm as it tries to refine the solutions at low residuals. What was so far seldom unappreciated is the influence of the undetermination of $\bar{\theta}$ on the other parameter and on $p_{\mathrm{v}}$. To understand Fig. 3 it is important to recall the influence of each parameter at different phase angle ranges. $B_{0}$ is only important close to the opposition. $\bar{\theta}$ has little effect at such low phase angles, but will become increasingly important at high phase angles. $\xi$ will have a significant influence on the integral phase function at both moderately low and moderately high solar phases (H93). Thus the undetermination of $\bar{\theta}$ plus its coupling with $\xi$ at moderate phase angles induces the spread of the latter; since $\xi$ is also coupled with $B_{0}$ at small phase angles this induces a spread of $B_{0}$. Finally, the spread in $p_{\mathrm{v}}$ due to the undetermination of $\bar{\theta}$ is caused mostly by the spreads of $\xi$ and $B_{0}$, since the direct influence of $\bar{\theta}$ on $p_{\mathrm{v}}$ is small. 


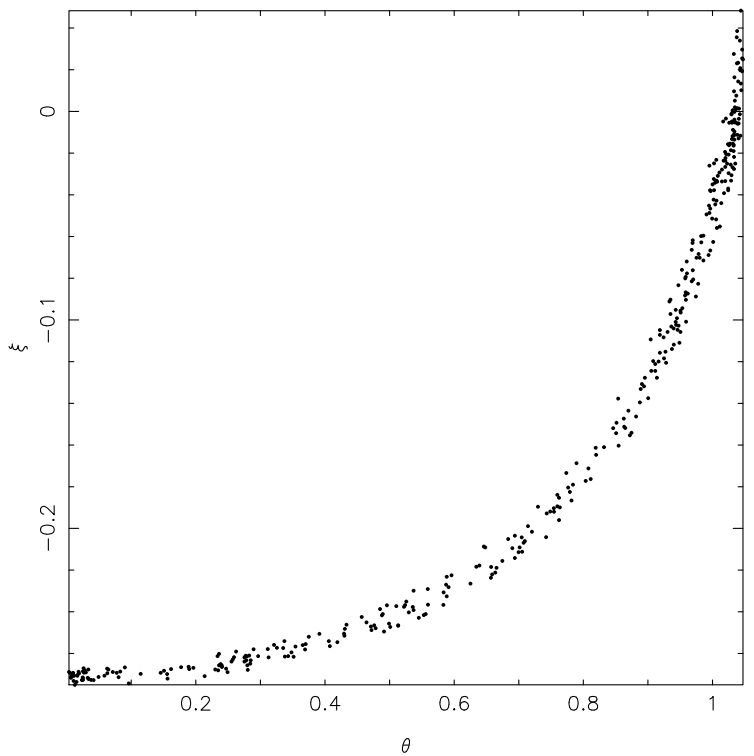

(a)

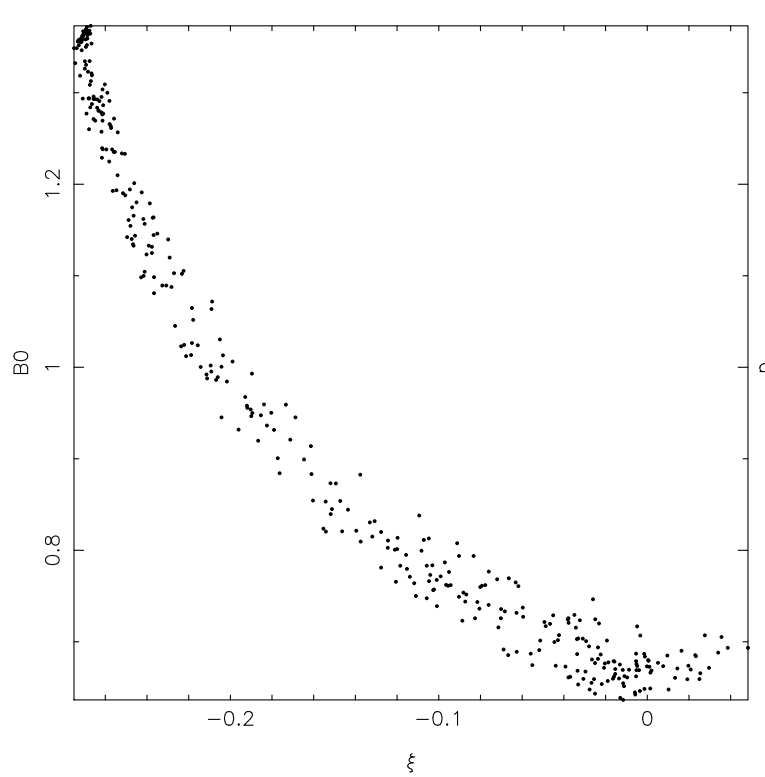

(c)

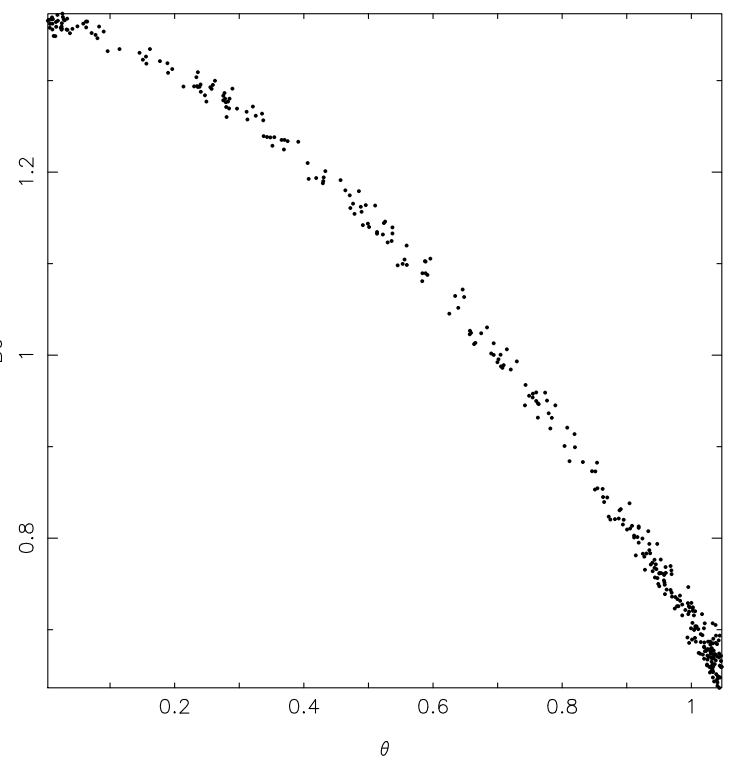

(b)

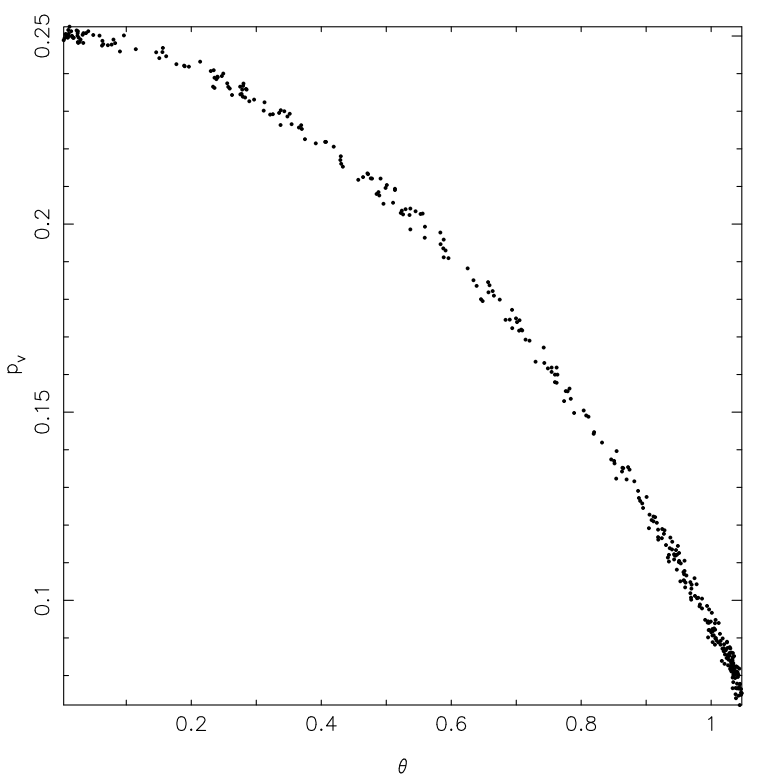

(d)

Fig. 3. Same results as Fig. 2, but showing the interdependece of the parameter in the solutions with residuals $<3.2 \times 10^{-3}$.

A similar behavior is observed for different combinations of $G$ and $w_{\mathrm{v}}$. However, the value that $\bar{\theta}$ assumes after it is forced to converge will depend on the given combination of $\left(G, w_{\mathrm{v}}\right)$. The significance of this is not readily clear.

A first result of the present work is that the undetermination of $\bar{\theta}$ precludes the existence of a single solution when trying to fit Hapke parameters to the Lumme-Bowell integral phase function, at least if only solar phase angles $\leq 60^{\circ}$ are considered. A consequence of this is that the scheme of Verbiscer \& Veverka for translating the HG system to Hapke parameters is only adequate for $\bar{\theta}=20^{\circ}$.

This also means that one trying to constrain the mineralogy of a body from its geometric albedo must take into account at least two other parameters: the slope parameter $G$ and the mean slope angle $\bar{\theta}$. The latter is notoriously difficult to determine. In principle it might be derived from photometric observations that extend to phase angles $>90^{\circ}$ or from disk-resolved observations (Domingue \& Hapke 1989; Helfenstein \& Veverka 1989). Although the present methodology is well suited to further study the existence of unique solutions for phase angles greater than $90^{\circ}$, Eq. (2) is only valid for $g<60^{\circ}$; for greater values of $g$ it would be necessary to interpolate the exact solutions of Eq. (4) (H93). Although feasible, this would be beyond the scope of this work and is left to a follow-up study. It is also possible to derive $\bar{\theta}$ from thermal (Müller 2002) and radar observations (Ostro et al. 1985). At any rate, the value this parameters is reliably known for only a handful of asteroids.

What can still be done at this point is to derive expressions for the scattering parameters $\left(B_{0}, h, \xi, w_{\mathrm{v}}\right)$ as functions of $p_{\mathrm{v}}, G$ and $\bar{\theta}$. This is an extension of the work of Verbiscer \& Veverka (1995), with the difference that here $\bar{\theta}$ is left as a free parameter. Then even if $\bar{\theta}$ is not known it would still be possible to limit the 


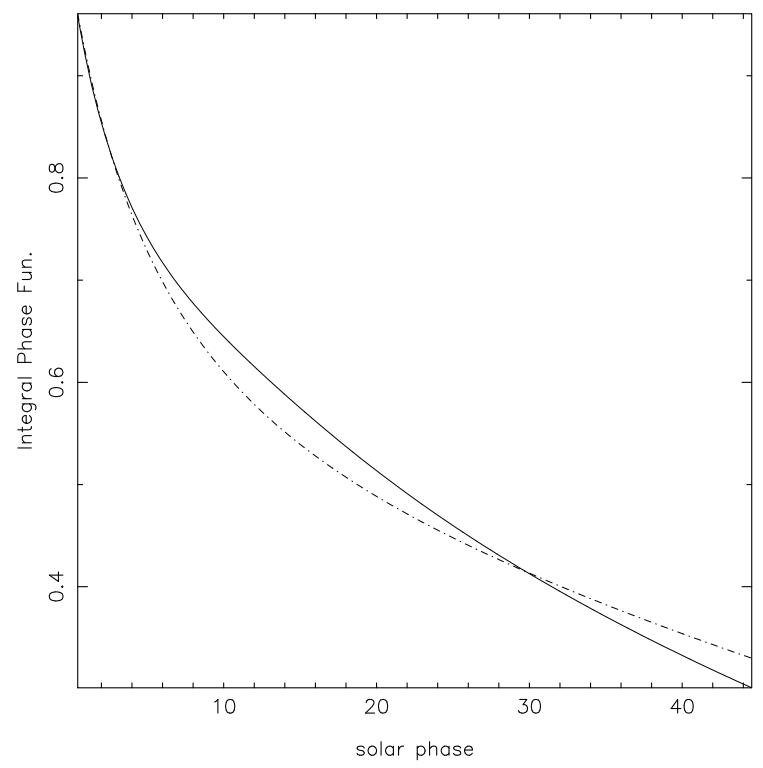

Fig. 4. Best fit of the Lumme-Bowell integral phase curve (Eq. (7), solid line) to the Hapke integral phase curve (Eq. (4), dashed) for laboratory scattering parameters of the $\mathrm{CV} 3$ sample with grain sizes in the $45-75 \mu \mathrm{m}$ range (see Table 2).

admissible range for the scattering parameters, $w_{\mathrm{v}}$ in particular. This would allow a compositional interpretation of the albedo, and would also be useful to estimate the scattering parameters to be used in attempts to fit spectra with radiative transfer models. Also, it would be valuable to have an expression to predict $p_{\mathrm{v}}$ for given values of $w_{\mathrm{v}}, G$ and $\bar{\theta}$, since this would allow to prospect for candidate asteroids with a composition characterized by a particular value of $w_{\mathrm{v}}$.

To accomplish this I performed a series of fits for $G \in$ $[-0.2,1]$, where $w$ and $\bar{\theta}$ were kept fixed and the best solutions for $h, \xi$ and $B_{0}$ were sought. I used $w \in[0,1]$ and $\bar{\theta} \in\left[0^{\circ}, 60^{\circ}\right]$, with steps of 0.04 for $G$ and $w$ and $6^{\circ}$ for $\bar{\theta}$. With the result of this it would be possible, following Verbiscer \& Veverka (1995), to derive the coefficients of polynomial fits to the adjusted parameters. That however proved to be neither practical nor accurate enough: it was necessary to consider polynomial with orders $>4$ and even so the result at some points differs from the exact value by more than $10 \%$. Instead, I used the results of the fits to assemble look-up tables which can then be interpolated numerically to any $(G, w, \bar{\theta})$ with good accuracy. I then used the resulting values of $w, \xi, B_{0}$ and $\bar{\theta}$ to assemble a look-up table for $p_{\mathrm{v}}$, using Eq. (1). This can be done since for a given triplet $(G, w, \bar{\theta})$ there is a unique value of $p_{\mathrm{v}}$. Similarly, It is also possible to obtain the scattering parameters $w, \xi, B_{0}$ and $h$ for given values of $\left(p_{\mathrm{v}}, G\right.$, $\bar{\theta})$.

\section{Application to asteroids and asteroid template materials}

In this section I compare the predicted geometric albedo of laboratory samples with the geometric albedo for a given $w$ as a function of $G$ and $\bar{\theta}$ that is obtained following the procedure presented in the previous section. Also, I compare both predictions with the measured values of $G$ and $p_{\mathrm{V}}$ of asteroids that have inferred composition similar to the laboratory samples.

The scattering parameters of a number of asteroid template materials measured in the lab can be found in the literature. These are usually determined (along with the other Hapke

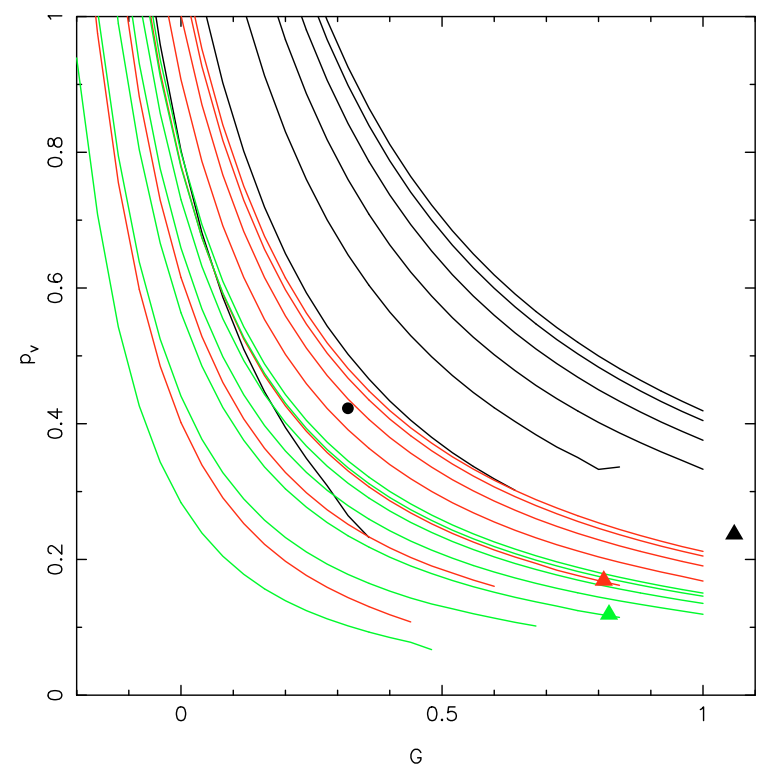

(a)

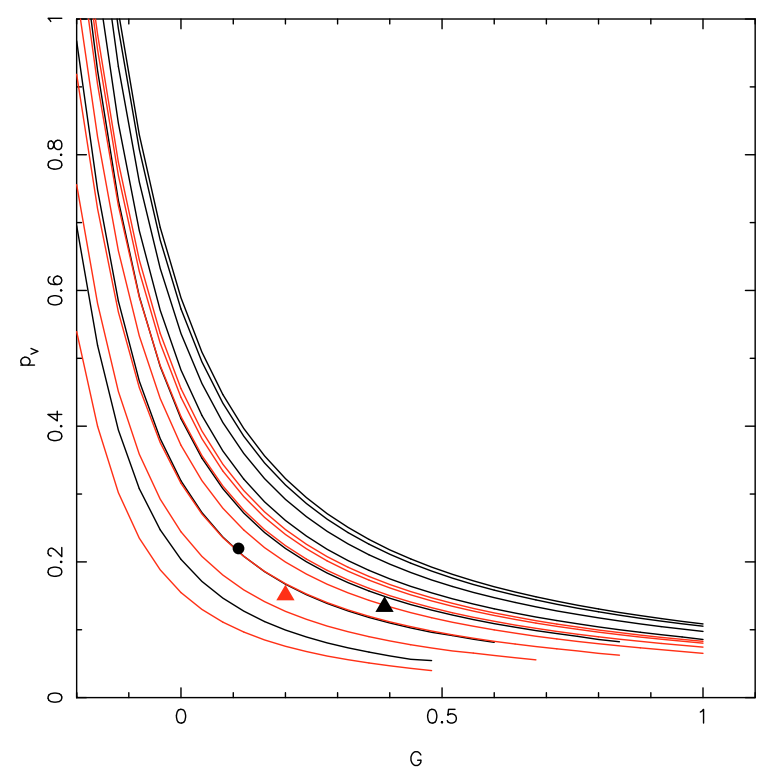

(b)

Fig. 5. Curves of the geometric albedo for a fixed $w_{\mathrm{v}}$ as a function of $G$ and $\bar{\theta}$. For each set of curves the lower one corresponds to $\bar{\theta}=60^{\circ}$ and the upper one to $\bar{\theta}=0^{\circ}$. a) Curves for the single scatter albedo of basalt samples for different grain sizes: $w=0.82$ (grains $<75 \mu \mathrm{m}$, in black), $w=0.57$ (75-250 $\mu \mathrm{m}$, red) and $w=0.45(250-500 \mu \mathrm{m}$, green). The observed values of $G$ and $p_{\mathrm{v}}$ for Vesta is shown as a black dot, while the values of $G$ and $P_{\mathrm{v}}$ obtained from the laboratory scattering parameters are shown as triangles, with the colors corresponding to the different grain sizes. b) Same for the CV3 sample. Black corresponds to $w=0.35$ (grain sizes 45-75 $\mu \mathrm{m}$ ) and red to $w=0.28(180-250 \mu \mathrm{m})$. The dot represents Lutetia.

scattering parameters) from the inversion of reflectance measurements of lab samples at several geometric configurations. Here we selected two samples: a telluric un-weathered basalt from Cord et al. (2003), and one CV3 carbonaceous chondrite (Allende) sample from Kamei \& Nakamura (2002). These will be compared, respectively with the observational parameters of the asteroids (4) Vesta and (21) Lutetia. Vesta is the only large basaltic asteroid in the Main Belt (McCord et al. 1970), 
Table 2. Scattering parameters of asteroid template material.

\begin{tabular}{lccccccccccc}
\hline \hline Sample & Size $(\mu \mathrm{m})$ & Wavelength $(\mu \mathrm{m})$ & $w$ & $\xi_{1}$ & $\xi_{2}$ & $f$ & $B_{0}$ & $h$ & $\bar{\theta}$ & $p_{\lambda}$ & $G_{\lambda}$ \\
\hline CV3 & $45-75$ & 514 & 0.35 & -0.04 & 0.58 & 0.26 & 2.2 & 0.06 & 6.0 & 0.134 & 0.39 \\
& $180-500$ & & 0.28 & -0.14 & 0.80 & 0.17 & 2.2 & 0.03 & 6.4 & 0.151 & 0.20 \\
Basalt & -75 & 559 & 0.82 & 0.49 & 0.13 & - & 0.15 & 0.24 & 5.8 & 0.237 & 1.06 \\
& $75-250$ & & 0.57 & 0.45 & 0.30 & - & 0.13 & 0.14 & 13.6 & 0.169 & 0.81 \\
& $250-500$ & & 0.45 & 0.41 & 0.32 & - & 0.16 & 0.14 & 16.2 & 0.119 & 0.82 \\
\hline
\end{tabular}

and the source of most of the basaltic HED meteorites (Cochran et al. 2004). Also, the reflectance spectrum of Vesta suggests that its surface has not been extensively affected by space weathering (Chapman 2004, Vernazza et al. 2006), which, among other things tends to lower the single scatter albedo of the exposed material. Several recent works based on spectra from the visible to the thermal infrared suggests that Lutetia is a likely candidate for a CV3-like composition (Birlan et al. 2006; Nedelcu et al. 2007) .

Table 2 shows the Hapke scattering parameter of the laboratory samples, the corresponding theoretical $p_{\mathrm{v}}$ for a spherical body and an estimation of the corresponding $G$. Note that the papers referenced in the table use different definitions of volumetric phase functions. While the geometric albedo is obtained directly using the scattering parameters in Eq. (1), deriving an estimation of the $G$ that more closely matches the scattering properties of the material is not so straightforward. To this end I generated a normalized integral phase function from the laboratory scattering parameters (using the adequate volumetric phase function) and attempted to fit it with the Lumme-Bowell empirical integral phase function. However, the best fit values found in general fail to adequately reproduce the theoretical integral phase functions: an example of the best match found is shown in Fig. 4. Therefore, a first result here is that scattering parameters derived in the lab will not necessarily produce an integral phase function that is compatible with what is seen in the asteroids (or, at least, with what is predicted by the Lumme-Bowell model).

Using the methods derived in the previous section it is then possible to obtain the possible values of the geometric albedo for the materials given in Table 2, and compare them to what is predicted from the laboratory scattering parameters and from the asteroids with presumed similar composition. Figure 5 shows the variation of the geometric albedo with the slope parameter for materials with different $w_{\mathrm{v}}$ and for given values of $\bar{\theta}$. Curves corresponding to each grain size range are represented in different colors. The laboratory derived values of $p_{\mathrm{v}}$ and $G$ and the values for the asteroids are also plotted. For each grain size the upper curve corresponds to $\bar{\theta}=0^{\circ}$ and the lower to $\bar{\theta}=60^{\circ}$, with intermediary curves at increments of $10^{\circ}$.

Considering the theoretical curves, the general trends are the same: for a given $w_{\mathrm{v}}$ the corresponding geometric albedo decreases with increasing $G$, and increasing $\bar{\theta}$ also decreases the geometric albedo. The effects of varying $\bar{\theta}$ are more pronounced for smaller $G$ and higher values of $w_{\mathrm{v}}$. Indeed, variations in $\bar{\theta}$ among asteroids with a same value of $w_{\mathrm{v}}$ can produce considerable diversity in the resulting geometric albedo. In Fig. 5a it can be seen that Vesta is compatible with unweathered basalt if we take either grains with $D<75 \mu \mathrm{m}$ with values of $\bar{\theta} \approx 55^{\circ}$ or with grains with $S 75-250 \mu \mathrm{m}$ and $\bar{\theta} \approx 25^{\circ}$, but for grains sizes in the $250-500 \mu \mathrm{m}$ range even the higher $\bar{\theta}$ would yield geometric albedos that are too low.

Considering Lutetia, its albedo and slope parameter can be matched by the sample of Allende with grain sizes in the $45-75 \mu \mathrm{m}$ range if we assume $\bar{\theta} \approx 50^{\circ}$ or with grains
$180-500 \mu \mathrm{m}$ for $\bar{\theta} \approx 40^{\circ}$. Finally, the values of the geometric albedo and the slope parameters derived from the scattering parameters measured in the lab tend to be very different from the asteroids with a presumed similar composition: in both cases we have too low geometric albedos and too high values for the slope parameter. This is particularly true for the basalt samples, but part of the discrepancy might come from the fact that the opposition parameters $\left(B_{0}, h\right)$ here were poorly constrained, since the measurements of Cord et al. (2003) were made for phase angles $>20^{\circ}$. The case of Lutetia is particularly interesting, since until recently the spectral evidence that it has a CV-like composition was at odds with the general perception that asteroids with carbonaceous chondrite compositions must necessarily have $p_{\mathrm{v}} \approx 0.1$ (i.e. Mueller et al. 2006).

\section{Conclusions}

In this work I attempted to analyze the effects of surface packing on the geometric albedo of asteroids, to provide a more consistent way to link the geometric albedo of asteroids to the single scatter albedo of laboratory samples and thus facilitate the use of the geometric albedo as a mineralogical discriminant.

I initially studied the relations between the slope parameter $G$ that describes the integral phase function of asteroids in the IAU HG system to the Hapke scattering parameters. It was found that the weak dependence of the integral phase function on the macroscopic roughness parameter $\bar{\theta}$ prevents the existence of a unique correspondence between a given slope parameter $G$ and the Hapke scattering parameters. Instead, I derived a numerical method to obtain the scattering parameters $\left(B 0, h, \xi, w_{\mathrm{v}}\right)$ as a function of $p_{\mathrm{v}}, G$ and $\bar{\theta}$ and also $\left(B 0, h, \xi, p_{\mathrm{v}}\right)$ as a function of $w_{\mathrm{v}}, G$, and $\bar{\theta}$. The numeric interpolation routines that perfom these transformations can be accessed at http: //funk . on . br/ c carvano/albedo/albedo. html.

Next it was shown that laboratory derived scattering parameters in general produce integral phase functions that are not compatible with what is expected from asteroids. This is probably due to the fact that the packing conditions on the surface of asteroids are very different from what can be simulated in the lab, and as a consequence the lab derived $\left(B 0, h, \bar{\theta}, \xi_{1}, \ldots\right)$ may not be representative of asteroid surfaces. Since $w$ in principle is not so strongly affected by the packing of the surface, I suggest that it is the only relevant scattering parameter that could derived in the lab. This also suggests that using a lab derived Hapke parameter when trying to fit asteroid spectra with radiative transfer models might not be accurate.

Finally, I showed that a given value of w can produce very different values of the geometric albedo depending on the values of $G$ and $\bar{\theta}$. Thus mineralogical inference based solely on geometric albedo can be misleading: asteroids with similar $p_{\mathrm{v}}$ may have very different $w_{\mathrm{v}}$, and vice-versa. Lutetia is a good example of how an incomplete appreciation of the factors that influence the geometric albedo may lead to confusion regarding the composition of asteroids. 
Acknowledgements. J. Carvano is supported by a fellowship from Conselho Nacional de Pesquisa Científica (CNPq).

\section{References}

Birlan, M., Vernazza, P., Fulchignoni, M., et al. 2006, A\&A, 454, 677 Bowell, E., Hapke, B., Domingue, D., et al. 1989, Asteroids II, 524

Carvano, J. M., Migliorini, A., Barucci, A., Segura, M., \& the CIRS Team 2007, Icarus, 187,574

Chapman, C. R. 2004, Ann. Rev. Ear. Plan. Sci., 32, 539

Clark, B. E., Bus, S. J., Rivkin, A. S., et al. 2004, J. Geophys. Res. (Planets), 109,2001

Cochran, A. L., Vilas, F., Jarvis, K. S., \& Kelley, M. S. 2004, Icarus, 167, 360

Cord, A. M., Pinet, P. C., Daydou, Y., \& Chevrel, S. D. 2003, Icarus, 165, 414

Domingue, D., \& Hapke, B. 1989, Icarus, 78, 330

Goldberg, D. E. 1989 (MA: Addison-Wesley)
Hapke, B. 1993, Topics in Remote Sensing (Cambridge, UK: Cambridge University Press)

Helfenstein, P., \& Veverka, J. 1989, Asteroids II, 557

Helfenstein, P., Veverka, J., Thomas, P. C., et al. 1994, Icarus, 107, 37

Holland, J. H. 1992 (Cambridge, MA: MIT Press)

Kamei, A., \& Nakamura, A. M. 2002, Icarus, 156, 551

McCord, T. B., Adams, J. B., \& Johnson, T. V. 1970, Science, 168, 1445

Mahfoud, S. W. 1995, Phd dissertation, Department of General Engeneering, University of Illinois at Urbana-Champaing

Müller, T. G. 2002, Meteoritics and Planetary Science, 37, 1919

Mueller, M., Harris, A. W., Bus, S. J., et al. 2006, A\&A, 447, 1153

Nedelcu, D. A., Birlan, M., Vernazza, P., et al. 2007, A\&A, 470, 1157

Ostro, S. J., Campbell, D. B., \& Shapiro, I. I. 1985, Science, 229, 442

Sakai, T., \& Nakamura, A. M. 2005, Earth, Planets, and Space, 57, 71

Tholen, D. J., \& Barucci, M. A. 1989, Asteroids II, 298

Verbiscer, A. J., \& Veverka, J. 1995, Icarus, 115, 369

Vernazza, P., Brunetto, R., Strazzulla, G., et al. 2006, A\&A, 451, L43 